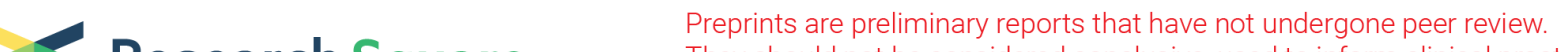 Research Square They should not be considered conclusive, used to inform clinical practice, or referenced by the media as validated information.
}

\section{Therapeutic Potential of Targeting Tfr/Tfh Cell Balance by Low-Dose-IL-2 in Active SLE: A Post-Hoc Analysis from a Double-Blind RCT Study}

\section{Miao Miao}

Peking University People's Hospital https://orcid.org/0000-0001-8927-4128

\section{Xian Xiao}

Peking University People's Hospital Department of Rheumatology and Immunology

Jiayi Tian

Peking University People's Hospital

\section{Yunzhi Zhufeng}

Peking University People's Hospital

\section{Ruiling Feng}

Peking University People's Hospital

\section{Ruijun Zhang}

Peking University People's Hospital

Jiali Chen

Peking University People's Hospital

\section{Xiaoying Zhang}

Peking University People's Hospital

\section{Bo Huang}

Peking University People's Hospital

\section{Yuebo Jin}

Peking University People's Hospital

\section{Xiaolin Sun}

Peking University People's Hospital

Jing He ( $\nabla$ hejing1105@126.com )

Peking University People's Hospital

\section{Zhanguo Li}

Peking University People's Hospital

\section{Research article}

Keywords: systemic lupus erythematosus, $T$ follicular helper cell, $T$ follicular regulatory cell, low-dose interleukin-2 
Posted Date: January 5th, 2021

DOl: https://doi.org/10.21203/rs.3.rs-138443/v1

License: (c) (i) This work is licensed under a Creative Commons Attribution 4.0 International License. Read Full License 


\section{Abstract}

Objective: To investigate the regulation of $\mathrm{T}$ follicular regulatory $(\mathrm{Tfr})$ and $\mathrm{T}$ follicular $(\mathrm{Tfh})$ cell subtypes by low-dose IL-2 in systemic lupus erythematosus (SLE) in a randomized, double-blind, placebo-controlled clinical trial.

Methods: A post-hoc analysis was performed in a randomized cohort of SLE patients $(n=60)$ receiving low-dose IL-2 therapy $(n=30)$ or placebo $(n=30)$, along with standard of care treatment. The primary end point was attainment of SLE responder index-4 (SRI-4) at week 12 in the trial. Twenty three healthy controls were enrolled for $T$ cell subsets detection at the same time with the trial. The t-stochastic neighbor embedding (tSNE) analysis of CD4 T subsets based on immune cells flow cytometry markers was performed to distinguish Tfh, Tfh1, Tfh2, Tfh17 and Tfr cell subsets.

Results: Compared with HC, the frequency of Tfr (CXCR5 ${ }^{+}$PD- $1^{\text {low }}$ Treg and CXCR5 ${ }^{+}$PD- ${ }^{\text {high }}$ Treg) cells was significantly reduced, while the pro-inflammatory Tfh cells were increased in patients with SLE. The imbalanced Tfh cell was associated with several pathogenic factors (anti-dsDNA antibodies $(r=0.309$, $P=0.027)$ and serum IL-17 ( $r=0.328, P=0.021))$ and SLE Disease Activity Index (SLEDAl) score $(r=0.273$, $\mathrm{P}=0.052)$. Decreased $C X C R 5^{+} \mathrm{PD}-1^{\text {low }}$ Treg/Tfh and $C X C R 5^{+} \mathrm{PD}-1^{\text {low }}$ Treg/Tfh17 were both associated with increased immunoglobulin $\mathrm{M}(\mathrm{IgM})(\mathrm{r}=-0.448, \mathrm{P}=0.002$ and $\mathrm{r}=-0.336, \mathrm{P}=0.024$ respectively). Efficacy of low-dose IL-2 therapy was associated with a restored Tfr/Tfh cell balance.

Conclusion: These data supports the hypothesis that promotion of Tfr associated with decreased disease activities, and that low-dose IL-2 therapy can recover Tfr/Tfh immune balance.

Trial registration number ClinicalTrials.gov Registries (NCT02465580).

\section{Key Messages}

1. Deregulation between $\mathrm{Tfr}$ and Tfh subsets associated with severity of SLE.

2. Low-dose IL-2 therapy was efficient in patients with SLE.

3. Low-dose IL-2 therapy elevates the Tfr/Tfh ratio, which might be a novel concept to design the therapeutic regimen.

\section{Introduction}

Systemic lupus erythematosus (SLE) is characterized by the breakdown of immune tolerance leading to auto-reactive immune responses and consequently, tissue and organ damages. Over the past decades, extensive studies on regulatory $\mathrm{T}$ (Treg) cells have revealed that these cells can maintain tolerance and regulate immune responses [1, 2], while T follicular helper cells (Tfh) play an important role in the production of autoantibodies and pro-inflammatory cytokines in SLE [3-5]. Moreover, the imbalance of the immune response between pro-inflammatory and anti-inflammatory cells is central in SLE pathogenesis. 
T follicular regulatory (Tfr) cells share features with Tfh and conventional Treg cells, and can inhibit Tfh cells and germinal center (GC) responses with a significant impact in humoral immunity [6-8]. Previous

studies have suggested that Tfr cells can be identified as CXCR5 ${ }^{+} \mathrm{PD}-1^{\text {low }}$ Treg and CXCR5 ${ }^{+} \mathrm{PD} 1^{\text {high }}$ Treg according to the surface marker CXCR5 and programmed cell death protein 1 (PD-1) [9-13]. The function of the two subsets of $\mathrm{Tfr}$ remains unclear. Imbalance or disfunction of Tfr subsets may directly or indirectly affect B cells, leading to expansion of overactive B cells which contributes to various immunerelated clinical diseases $[14,15]$. So far, the role of balance between Tfh and Tfr subsets in SLE is still controversial due to the heterogeneity of the disease, cohort size, and methods of studies $[16,17]$.

Efficacious treatments, including low-dose Interleukin 2 (IL-2), might promote Tfr cell responses, and inhibit Tfh cell development in SLE $[18,19]$. However, it is not well understood how these circulating Tfhlike cell subsets, including Tfr and Tfh subsets, are involved in the disease. To date, the balance of these new subsets has not been addressed, neither how these cells respond to SLE treatment. Here, we identified an imbalanced profile of Tfh cell subsets in SLE, including newly described anti-inflammatory CXCR5 ${ }^{+}$PD-1 ${ }^{\text {low }}$ Treg, CXCR5 ${ }^{+}$PD- $1^{\text {high }}$ Treg and the pro-inflammatory Tfh and Tfh 17 ; and investigated the change of these subtypes by low-dose IL-2 treatment in a randomized, double-blind, placebo-controlled study in SLE.

\section{Participants And Methods}

\section{Participants}

This was a post-hoc analysis of data from an RCT clinical study (NCT02465580) of low-dose IL-2 in SLE patients. Full details of study designs and inclusion/exclusion criteria for each completed study have previously been published [1, 2]. Studies were conducted in accordance with the Declaration of Helsinki, the International Conference on Harmonization Guidelines for Good Clinical Practice. Besides, 23 healthy controls (HC) were enrolled during the same time of RCT study. Table 1 summarized baseline characteristics. Written informed consents were obtained from these healthy controls. The experimental protocol followed the guidelines of the Declaration of Helsinki and was approved by the Human Ethics Committee of Peking University People's Hospital (Beijing, China). 
Table 1

Baseline characteristics of SLE patients and healthy controls $(\mathrm{HC})$ in this study

\begin{tabular}{|llll|}
\hline Characteristics & SLE $(\mathbf{n}=60)$ & HC $(\mathbf{n = 2 3})$ & Pvalue \\
\hline Age, year, mean \pm SD & $30.84 \pm 9.48$ & $29.83 \pm 9.72$ & 0.474 \\
\hline Female/Male & $56 / 4$ & $21 / 2$ & $>0.99$ \\
\hline Duration, months, mean \pm SD & $65.15 \pm 58.65$ & - & - \\
\hline Medications & & - & - \\
\hline Prednisone dose, mg/day, median (range) & $13.5(0,50)$ & - & - \\
\hline Hydroxychloroquine & $57(95)$ & - & - \\
\hline Cyclophosphamide & $4(6.67)$ & - & - \\
\hline Azathioprine & $5(8.33)$ & - & - \\
\hline Cyclosporine & $5(8.33)$ & - & - \\
\hline Mycophenolate Mofetil & $17(28.33)$ & - & - \\
\hline Tacrolimus & $2(3.33)$ & - & - \\
\hline Leflunomide & $4(6.67)$ & - & - \\
\hline Thalidomide & $1(1.67)$ & - & - \\
\hline Methotrexate & $1(1.67)$ & - & - \\
\hline Interleukin-2 & $30(50)$ & - & - \\
\hline $\begin{array}{l}\text { For a continuous variable, median (range) or mean } \pm \text { SD. For a categorical variable, count } \\
\text { (percentage). SLE, Systemic lupus erythematosus. }\end{array}$ & & \\
\hline
\end{tabular}

\section{Flow Cytometric Analysis}

Single-cell suspensions from peripheral blood in SLE patients and HC were analyzed by multicolor flow cytometry (FACSAria II; BD Biosciences, Franklin Lakes, NJ, USA). t-stochastic neighbor embedding (tSNE) analysis of CD4 T subsets based on immune cells flow cytometry markers was shown in Fig. 1. Data were also analyzed using FlowJo v10 software (Tree Star, Ashland, OR, USA) (Figure S1). The absolute number of CD4 T cell subsets was calculated by multiplying proportion of CD 4 T cell subsets in lymphocytes by absolute lymphocyte number determined with an automated hematology analyzer. Detailed protocol of trial has been published online [18].

\section{Cytometric Beads Array (cba) Analysis Of Serum Cytokines}


Serum levels of IL-2, IL-17 and other inflammatory cytokines were determined by human Th1/Th2/Th17 14-plex (QuantoBio, Beijing, China) according to the manufacturer's instructions.

\section{Statistical analysis}

Data were expressed as the median and range for non-normally distributed data, while mean \pm standard deviation (SD.) for normally distributed data. The Student's unpaired or paired $t$ test was performed to compare two groups for parametric data, and the Mann-Whitney $\mathrm{U}$ test or Wilcoxon rank sum test was performed for nonparametric data. Relationships between variables were analyzed by Spearman's rank test. Statistical analyses were performed using SPSS v.22.0 or R v.3.6.3 software. Two-sided P values < 0.1 were considered statistically significant.

\section{Results}

\section{Characteristics of SLE patients}

Given recent studies showing the imbalances in the effector and regulatory Tfh cells compartment in SLE patients [3, 4], and in light of our finding that low-dose IL-2 treatment significantly influences Tfh subtypes, we recruited a small cohort of healthy controls $(H C)(n=23$, Table 1$)$ for comparative analysis.

The demographic and clinical manifestations of these patients were shown in Table 1. There was no significant difference between patients and HCs regarding age or gender. 98\% of the SLE patients were positive in anti-dsDNA tests, $42 \%$ had renal involvement and $48 \%$ had skin manifestations.

\section{Imbalanced Tfr/Tfh in SLE}

As shown in Table 2, regulatory T cells including Treg, CXCR5 ${ }^{+} P D-1^{\text {low }}$ Treg, CXCR5 ${ }^{+} P D-1^{\text {high }}$ Treg cells were significantly reduced in SLE patients than those in $\mathrm{HC}(P=0.087, P=0.033, P<0.001, P<0.001$, respectively). In contrast, the effector Tfh cells were significantly increased in SLE patients than in HC $(p=0.081)$. Besides, $T f r$ subsets: Tfh subsets ratios in SLE were dramatically decreased, including CXCR5 ${ }^{+}$PD- $1^{\text {low }}$ Treg/Tfh (P=0.043), CXCR5 ${ }^{+}$PD- $1^{\text {high }}$ Treg/Tfh $(p<0.001), C X C R 5^{+}$PD- ${ }^{\text {low }}$ Treg/Tfh17 $(P=0.052)$ and $C X C R 5^{+} P D-1^{\text {high }}$ Treg/Tfh17 $(P<0.001)$.

\section{High Tfh associated with decreased serum IL-2}

As show in Figure 2, an expanded inflammatory Tfh cell compartment was correlated with higher serum IL-17 levels $(r=0.328, P=0.021)$ and increased frequency of Tfh17 correlated with lower serological IL-2 $(r=-0.295, P=0.04)$ (Figure 2). There was a reduction of CXCR5/PD-1 double positive subset (CXCR5 ${ }^{+}$PD $1{ }^{\text {high }}$ Treg), which was related with IL-10 elevation ( $r=0.243, P=0.093$, Figure 3 ). 
Table 2. Difference of CD4 T subsets between HC and SLE, and between active and remission group.

Variables

$$
\begin{aligned}
& \mathrm{HC} \\
& (\mathrm{n}=23)
\end{aligned}
$$

SLE

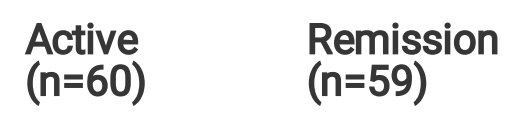

$P$,

HC VS.

$P$, Remission

VS. Active

(Before (After

therapy)

therapy)

Proportion (percentage in lymphocyte, \%)

$\begin{array}{llllll}\text { Treg } & 1.22(1,1.47) & \begin{array}{l}0.95(0.65, \\ 1.53)\end{array} & \begin{array}{l}1.6(0.97, \\ 2.49)\end{array} & 0.087 & 0.001 \\ & & & & \\ \text { CXCR5'PD- } & 0.09(0.04, & \begin{array}{l}0.06(0.03, \\ 0.1)\end{array} & \begin{array}{l}0.13(0.06, \\ 0.25)\end{array} & 0.033 & <0.001 \\ \text { 1lowTreg } & 0.14) & & & \end{array}$

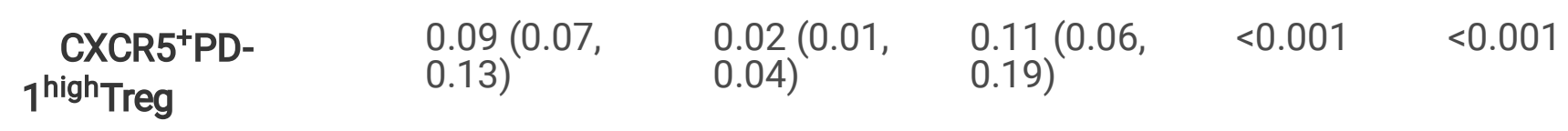

\begin{tabular}{clllll} 
Tfh & $0.28(0.17$, & $0.39(0.24$, & $0.19(0.09$, & 0.081 & 0.002 \\
& $0.43)$ & $0.56)$ & $0.37)$ & & \\
Tfh1 & $0.61(0.38$, & $0.2(0.08$, & $0.17(0.09$, & $<0.001$ & 0.42 \\
& $0.99)$ & $0.36)$ & $0.28)$ & & \\
Tfh2 & $1.06(0.58$, & $0.42(0.21$, & $0.36(0.19$, & $<0.001$ & 0.367 \\
& $1.5)$ & $0.6)$ & $0.68)$ & & \\
Tfh17 & $0.45(0.15$, & $0.47(0.27$, & $0.44(0.2$, & 0.345 & 0.851 \\
& $0.65)$ & $0.87)$ & $0.83)$ & & \\
& & & & & \\
\hline
\end{tabular}

\begin{tabular}{|c|c|c|}
\hline Treg & $\begin{array}{l}25.51 \text { (19.98, } \\
30.78)\end{array}$ & $\begin{array}{l}11.94(6, \\
20.81)\end{array}$ \\
\hline
\end{tabular}

Absolute number (cells/L)

\begin{tabular}{|c|c|c|c|c|c|}
\hline $\begin{array}{l}\text { CXCR5 }{ }^{+} \text {PD- } \\
\text { 1 }^{\text {high }} \text { Treg }\end{array}$ & $\begin{array}{l}2.99(1.18, \\
3.57)\end{array}$ & $\begin{array}{l}0.24(0.06, \\
0.56)\end{array}$ & $\begin{array}{l}1.5(0.71, \\
2.27)\end{array}$ & 0.001 & $<0.001$ \\
\hline
\end{tabular}

$\begin{array}{rlllll}\text { CXCR5 }{ }^{+} \text {PD- } & 2.98(1.71, & 0.67(0.24, & 1.33(0.8, & 0.037 & 0.005 \\ \text { 1 }^{\text {low }} \text { Treg } & 3.1) & 1.21) & 3.54) & & \end{array}$

$\begin{array}{clllll}\text { Tfh } & \begin{array}{l}7.29(4.34, \\ 8.92)\end{array} & \begin{array}{l}4.6(2.58, \\ 10.28)\end{array} & \begin{array}{l}2.84(0.81, \\ 6.04)\end{array} & 0.625 & 0.008 \\ & 14.26(12.37, & \begin{array}{l}2.11(0.95, \\ \text { Tfh1 }\end{array} & \begin{array}{l}1.92(0.68, \\ 4.28)\end{array} & 0.002 & 0.427 \\ & 18.22) & 5.47) & & \\ \text { Tfh2 } & 19.31(14.66, & 4.43(2.11, & 4.04(1.73, & 0.002 & 0.751 \\ & 24.22) & 11.2) & 11.88) & & \\ \text { Tfh17 } & 8.82(7.25, & 5.39(2.33, & 4.67(2.47, & 0.642 & 0.664 \\ & 12.22) & 14.38) & 10.36) & & \\ & & & & & \\ \end{array}$




\section{Ratios}

\begin{tabular}{|c|c|c|c|c|c|}
\hline Treg/Tfh & $\begin{array}{l}3.69(3.04, \\
8.49)\end{array}$ & $\begin{array}{l}2.49(1.89, \\
4.5)\end{array}$ & $\begin{array}{l}8.93(4.04, \\
18.29)\end{array}$ & 0.002 & $<0.001$ \\
\hline $\begin{array}{l}\text { CXCR5+PD- } \\
\text { 1 }^{\text {lowTreg/Tfh }}\end{array}$ & $\begin{array}{l}0.3(0.21 \\
0.57)\end{array}$ & $\begin{array}{l}0.16(0.08, \\
0.31)\end{array}$ & $\begin{array}{l}0.61(0.4 \\
1.32)\end{array}$ & 0.002 & $<0.001$ \\
\hline $\begin{array}{c}\text { CXCR5+PD- } \\
1^{\text {high }} \text { Treg/Tfh }\end{array}$ & $\begin{array}{l}0.46(0.21, \\
0.69)\end{array}$ & $\begin{array}{l}0.05(0.01, \\
0.1)\end{array}$ & $\begin{array}{l}0.54(0.24 \\
1.3)\end{array}$ & $<0.001$ & $<0.001$ \\
\hline Treg/Tfh17 & $\begin{array}{l}3.87(2.27, \\
5.71)\end{array}$ & $\begin{array}{l}2.29(1.23, \\
4.21)\end{array}$ & $\begin{array}{l}3.89 \text { (2.01, } \\
6.62)\end{array}$ & 0.047 & $<0.001$ \\
\hline $\begin{array}{c}\text { CXCR5+PD- } \\
\text { 1 }^{\text {low }} \text { Treg/Tfh17 }\end{array}$ & $\begin{array}{l}0.25(0.17 \\
0.34)\end{array}$ & $\begin{array}{l}0.11(0.05 \\
0.22)\end{array}$ & $\begin{array}{l}0.28(0.18, \\
0.52)\end{array}$ & 0.002 & $<0.001$ \\
\hline $\begin{array}{c}\text { CXCR5 }{ }^{+} \text {PD- } \\
\text { 1 }^{\text {high }} \text { Treg/Tfh17 }\end{array}$ & $\begin{array}{l}0.3(0.14 \\
0.64)\end{array}$ & $\begin{array}{l}0.03(0.01, \\
0.09)\end{array}$ & $\begin{array}{l}0.23(0.12, \\
0.45)\end{array}$ & $<0.001$ & $<0.001$ \\
\hline
\end{tabular}

SLE, systemic lupus erythematosus. HC, healthy controls. Data are median (IQR).

\section{Imbalanced Tfh and Tfr cell association with disease activity in SLE}

Treg cells were decreased and associated with elevated ESR $(r=-0.382, \mathrm{P}<0.01)$ and Safety of Estrogens in Lupus Erythematosus National Assessment version of the SLE Disease Activity Index (SLEDAI) $(r=-0.245$, $P=0.089$, Figure 2). At the same time, the decrease of $C X C R 5^{+} P D-1^{\text {low }}$ Treg was associated with increased ESR and anti-dsDNA antibodies production (Figure 2, 3). In contrast, an increased inflammatory Tfh cell compartment was found and was correlated with elevated SLEDAl, titer of anti-AnuA, anti-dsDNA antibodies, serum IL-17 and decreased C3 (Figure 2, 3).

Upon further analysis of the correlation between regulatory and effector subsets, we found decreased Treg/Tfh ratio in severe patients with higher SLEDAI score, higher titers of anti-AnuA and anti-dsDNA antibodies (Figure 3). In addition, there was a reduced CXCR5 ${ }^{+}$PD $1^{\text {low }}$ Treg/Tfh in this group of severe patient.

Figure 3 showed that the frequency of Tfh1 and Tfh2 was positively correlated with the number of total B cells and switched memory $B\left(\mathrm{CD}_{19}{ }^{+} \mathrm{IgD} \mathrm{CD}^{-} 7^{+}\right)$cells. CXCR5 ${ }^{+} \mathrm{PD}-1^{\text {low }}$ Treg/Tfh 17 was negatively correlated with switched memory $B$ cells and plasma $B$ cells $(r=-0.341, P=0.027$, Figure 3$)$. And decreased CXCR5 ${ }^{+}$PD- $1^{\text {low }}$ Treg/Tfh and CXCR5 ${ }^{+}$PD- $1^{\text {low }}$ Treg/Tfh17 were both associated with increased serum level of IgM.

\section{Low-dose IL-2 therapy increased Tfr/Tfh ratio in SLE patients}

In this RCT of low-dose IL-2 therapy in SLE, low-dose IL-2 significantly increased Tregs [10]. With effective treatment, British Isles Lupus Assessment Group (BILAG), SLEDAI, SLE Responder Index-4 (SRI-4), 
physician's global assessment (PGA), myositis, fever, alopecia, vasculitis, arthritis, oral ulcer and rash were all improved at week 12 (Figure 4) [10]. After 3 cycles of low-dose IL-2 therapy, the frequency of CXCR5 ${ }^{+}$PD- $1^{\text {low }}$ Treg cells and CXCR5 ${ }^{+}$PD- ${ }^{\text {high }}$ Treg cells in lymphocyte was significantly increased at week 12 compared to placebo control $(0.06(0.03,0.1)$ vs. $0.13(0.06,0.25), p<0.001$ and $0.02(0.01,0.04)$ vs. $0.11(0.06,0.19), P<0.001$ respectively) (Table 2, Figure 4). Similarly, the absolute number of these Treg cells (CXCR5 ${ }^{+} \mathrm{PD}-1^{\text {high }}$ Treg and $\mathrm{CXCR} 5^{+} \mathrm{PD}-1^{\text {low }}$ Treg) were significantly increased after the treatment of low-dose IL-2 $(0.67(0.24,1.21)$ vs. $1.33(0.8,3.54), P=0.005$ and $0.24(0.06,0.56)$ vs. $1.5(0.71,2.27)$, $\mathrm{P}<0.001$ respectively) (Table 2, Figure 4). Besides, compared to baseline, Tfr subsets: Tfh subsets ratios in SLE were dramatically increased, including CXCR5 ${ }^{+} \mathrm{PD}-1^{\text {low }}$ Treg/Tfh $(\mathrm{P}<0.001), \mathrm{CXCR} 5^{+} \mathrm{PD}-$ $1^{\text {high }}$ Treg/Tfh $(p<0.001), C X C R 5^{+} P D-1^{\text {low }}$ Treg/Tfh17 $(P<0.001)$ and CXCR5 ${ }^{+}$PD- ${ }^{\text {high }}$ Treg/Tfh17 $(\mathrm{P}<0.001)$.

\section{Discussion}

Increasing evidence indicates that Tfh cells are important in the pathogenesis of SLE. Tfh cells are recognized as a distinct T-cell subset, which provides help for GC formation, B-cell affinity maturation, and immunoglobulin class switching, as an indispensable part of adaptive immunity. Our previous work showed that patients with SLE have an increased number of peripheral Tfh cells, which positively correlates with autoantibody titers (anti- dsDNA antibodies) and disease activity, as measured by the SLEDAl. Others have reported that the aberrant expression of Tfh cells is a common feature in mouse models of SLE, suggesting its contribution in the development of autoimmune diseases $[4,5]$.

Besides Tfh cells, a subset of Treg cells, named Tfr cells, have been identified. These cells share common characteristics with Tfh and conventional Treg cells, and can inhibit GC responses, regulating the number of Tfh and GC B cells [6-8]. Therefore, it is generally believed that Tfr cells constrain the B-cell "help" provided by Tfh cells to maintain immune homeostasis. An aberrant or disordered Tfh/ Tfr balance may result in the break of tolerance, excessive B-cell proliferation, antibody production, and the development of autoimmune diseases. A recent study showed the importance of the Tfr/ Tfh balance in autoimmune responses in BXD2 mice, which display spontaneous autoreactive GC formation [21]. In addition, intravenous immunoglobulin administration to mice with collagen-induced arthritis augments the number of Tfr cells and represses the subsequent maturation of GC B cells [22], which also supportes the idea of a critical role for Tfr cells in autoimmune diseases.

In our study, we found a deficiency of Tfr cell subsets, including CXCR5 ${ }^{+}$PD- ${ }^{\text {low }}$ Treg, and CXCR5 ${ }^{+}$PD$1^{\text {high }}$ Treg; and increased Tfh cells in the peripheral blood of SLE patients. The shifted balance between circulating CXCR5 ${ }^{+}$PD- ${ }^{1 \text { low }}$ Treg and Tfh cells correlated not only with reduced serum IL-2, IL-10 and increased IL-21 levels in patients; but also with clinical SLE parameters, e.g. ESR, anti-dsDNA antibodies and disease activity (SLEDAI Scores). These findings are consistent with previous studies in vitro, in which Tfh and CXCR5 ${ }^{+} \mathrm{PD}-1^{\text {low }}$ Treg or $\mathrm{CXCR} 5^{+} \mathrm{PD} 1^{\text {high }}$ Treg cells can antagonize $\mathrm{B}$ cell function, production of high-affinity antibodies and the memory B cell differentiation [22]. CXCR5 ${ }^{+}$PD-1 ${ }^{\text {low }}$ Treg cells 
play an important immunosuppressive function by curbing self-reactive auto-antibodies development within the GC during an inflammatory immune response [5]. Therefore, deregulation of the Tfr and Tfh cell compartments is associated with disease severity, B cell frequency and antibody production in SLE.

There have been several relatively successful attempts to reduce the severity of SLE in humans via blockade of Tfh-cell differentiation and activity. Studies using monoclonal antibodies against ICOSL inhibited the development of Tfh and GC B cells resulting in decreased anti-dsDNA antibodies and improved kidney function in both human and mouse [23]. For years, SLE therapy has relied on broad spectrum immunosuppressants; however, a growing body of work shows that a targeted increase of regulatory $T$ cells may be a more attractive therapy $[18,19,24,25]$.

IL-2 is essential for the development and maintenance of Treg cells, which prevent the development of autoimmune disease. Low-dose IL-2 can promote Tregs by activating the transcription factor STAT5, which binds to the Foxp3 locus and promotes Foxp3 expression without activation of effector T cells. More recently, IL-2 has been shown to be essential for the inhibition of Tfh cell development. Thus, in this study, we asked if low-dose IL-2 therapy might also elevate the Tfr/Tfh ratio, exploring a novel concept for rational therapeutic design.

Our previous studies had proven a deficient Treg cell compartment and decreased IL-2 levels in circulation of SLE, and the efficacy of low-dose IL-2 treatment. But there was no study addressing the impact of lowdose IL-2 on Tfr : Tfh balance. After effective therapy, especially low-dose IL-2 therapy, the imbalanced Tfr and Tfh subsets were reversed accompanying improvement of disease activity. Furthermore, Tfr subsets were all increased regardless of output measurement; proportion and absolute number. Although we didn't see a significant change in Th17 frequency, the ratios of CXCR5 ${ }^{+}$PD- ${ }^{\text {low }}$ Treg/Tfh 17 and CXCR5 ${ }^{+}$PD- $1^{\text {high }}$ Treg/Tfh 17 were significantly decreased compared to those in healthy controls. Besides, we didn't see any obvious difference between CXCR5 ${ }^{+}$PD- ${ }^{\text {low }}$ Treg and CXCR5 ${ }^{+}$PD $-1^{\text {high }}$ Treg, perhaps reflecting a functional overlap of these two subsets.

\section{Conclusions}

In summary, our findings indicate that imbalance of Tfh and Tfr is important for SLE severity; and lowdose IL-2 ameliorates lupus autoimmunity favoring Tfr cell expansion. Our study added to these findings by demonstrating that low-dose IL-2 therapy selectively activates and expands Tfr cells, while demonstrating clinical efficacy in SLE. Further studies are needed to better understand how to explore Tfh cell or Tfr cell signatures to stratify patients, and guide the design of novel treatment regiments for SLE in future clinical trials.

\section{Abbreviations}

Tfr: T follicular regulatory; Tfh: T follicular; IL-2: interleukin-2; SLE: systemic lupus erythematosus; tSNE: tstochastic neighbor embedding; HC: healthy controls; IL-17: interleukin-17; SLEDAI: SLE Disease Activity 
Index; IgM: immunoglobulin M; Treg: regulatory T; GC: germinal center; SD: standard deviation; SRI-4: SLE responder index-4; Anti-AnuA: anti-nucleosome antibody; Anti-dsDNA: anti-double-stranded DNA antibody; C3: complement 3; BILAG: British Isles Lupus Assessment Group; PGA: physician's global assessment; ESR: erythrocyte sedimentation rate. PD-1: programmed cell death protein 1.

\section{Declarations}

\section{Acknowledgements}

We acknowledge the patients, research nurses and clinicians who have helped support this study.

\section{Author's contributions}

$\mathrm{JH}$ had full access to all of the data in the study and takes responsibility for the integrity of the data and the accuracy of the data analysis. JH, XS, ZL and MM conceived of and designed the study. MM, XX, JT, $Y Z, R Z, R F, J C, X Z, B H$ and $Y J$ acquired the data. $M M, X X, J T, Y Z$ and $R F$ analyzed and interpreted the data. All authors were involved in drafting the article or making critical revisions for important intellectual content, and all authors read and approved the submitted manuscript.

\section{Funding}

The National Natural Science Foundation of China $(31870879,81971520)$, Peking-Tsinghua Center for Life Sciences, Peking University Clinical Scientist Program (BMU2019LCKXJ004) and Clinical Medicine Plus X-Young scholars Project of Peking University (PKU2020LCXQ018) supported by the Fundamental Research Funds for the Central Universities.

\section{Ethics approval and consent to participate}

This study was approved the Human Ethics Committee of Peking University People's Hospital (Beijing, China) and all subjects provided written informed consent.

\section{Consent for publication}

Not applicable.

\section{Disclosure statement}

The authors have declared no conflicts of interest.

\section{Data availability statement}

Data are available upon reasonable request.

\section{Author details}


${ }^{1}$ Department of Rheumatology \& Immunology, Peking University People's Hospital, Beijing, 100044, China.

${ }^{2}$ Beijing Key Laboratory for Rheumatism Mechanism and Immune Diagnosis (BZ0135).

\section{References}

1. Sakaguchi S, Yamaguchi T, Nomura T, Ono M. Regulatory T cells and immune tolerance. Cell. 2008;133:775-87.

2. Yuan X, Cheng G, Malek TR. The Importance of Regulatory T-cell Heterogeneity in Maintaining SelfTolerance. Immunol Rev. 2014;259(1):103-14.

3. Papp G, Szabó K, Szekanecz Z, Zeher M. Follicular helper T cells in autoimmune diseases. Rheumatology. 2014;53:1159-60.

4. Simpson N, Gatenby P, Wilson A, Malik S, Fulcher D, Tangye S, et al. Expansion of circulating T cells resembling follicular helper $T$ cells is a fixed phenotype that identifies a subset of severe systemic lupus erythematosus. Arthritis Rheum. 2010;62:234-44.

5. Akiyama M, Suzuki K, Yamaoka K. Number of Circulating Follicular Helper 2 T Cells Correlates With IgG4 and Interleukin-4 Levels and Plasmablast Numbers in IgG4-Related Disease. Arthritis Rheumatol. 2015;67(9):2476-81.

6. Chung Y, Tanaka S, Chu F, Nurieva RI, Martinez GJ, Rawal S, et al. Follicular regulatory T cells expressing Foxp3 and Bcl-6 suppress germinal center reactions. Nat Med. 2011;17:983-8.

7. Linterman MA, Pierson W, Lee SK, Kallies A, Kawamoto S, Rayner TF, et al. Foxp3 + follicular regulatory T cells control the germinal center response. Nat Med. 2011;17:975-82.

8. Wollenberg I, Agua-Doce A, Hernández A, Almeida C, Oliveira VG, Faro J, et al. Regulation of the germinal center reaction by Foxp3 + follicular regulatory T cells. J Immunol. 2011;187:4553-60.

9. Sayin I, Radtke AJ, Vella LA, Jin W, Wherry EJ, Buggert M, et al. Spatial distribution and function of T follicular regulatory cells in human lymph nodes. J Exp Med. 2018;215:1531-42.

10. Hatam LJ, Devoti JA, Rosenthal DW, Lam F, Abramson AL, Steinberg BM, et al. Immune suppression in premalignant respiratory papillomas: enriched functional CD4 + Foxp3 + regulatory T cells and PD1/PD-L1/L2 expression. Clin Cancer Res. 2012;18:1925-35.

11. Ritvo PG, Churlaud G, Quiniou V, Florez L, Brimaud F, Fourcade G, et al. T cells lack IL-2Ra but express decoy IL-1R2 and IL-1Ra and suppress the IL-1-dependent activation of T cells. Sci Immunol. 2017;215:eaan0368.

12. Zhou Q, Munger ME, Highfill SL, Tolar J, Weigel BJ, Riddle M. Program death-1 signaling and regulatory $\mathrm{T}$ cells collaborate to resist the function of adoptively transferred cytotoxic $\mathrm{T}$ lymphocytes in advanced acute myeloid leukemia. Blood 2010 Oct 07;116:2484-93.

13. Maeda T, Wakasawa T, Shima Y, Tsuboi I, Aizawa S, Tamai I. Role of polyamines derived from arginine in differentiation and proliferation of human blood cells. Biol Pharm Bull. 2006;292:2186- 
97.

14. Fu W, Liu X, Lin X, Feng H, Sun L, Li S, et al. Deficiency in T follicular regulatory cells promotes autoimmunity. J Exp Med. 2018;215:815-25.

15. Sage PT, Alvarez D, Godec J, von Andrian UH, Sharpe AH. Circulating T follicular regulatory and helper cells have memory-like properties. J Clin Invest. 2014;124:5191-204.

16. Xu B, et al. The ratio of circulating follicular $\mathrm{T}$ helper cell to follicular $\mathrm{T}$ regulatory cell is correlated with disease activity in systemic lupus erythematosus. Clin Immunol. 2017;183:46-53.

17. Liu C, et al. Increased circulating CD4 + CXCR5 + FoxP3 + follicular regulatory T cells correlated with severity of systemic lupus erythematosus patients. Int Immunopharmacol. 2018;56:261-8.

18. He J, Zhang R, Shao M, et al. Efficacy and safety of low-dose IL-2 in the treatment of systemic lupus erythematosus: a randomised, double-blind, placebo-controlled trial. Ann Rheum Dis. 2020;79(1):141-9.

19. He J, Zhang $X$, Wei $Y$, Sun $X$, Chen $Y$, et al. Low-dose interleukin-2 treatment selectively modulates CD4 + T cell subsets in patients with systemic lupus erythematosus. Nat Med. 2016;22:991-3.

20. Furie RA, Petri MA, Wallace DJ, et al. Novel evidence-based systemic lupus erythematosus Responder index. Arthritis Rheum. 2009;61:1143-51.

21. Ding Y, Li J, Yang P, Luo B, Wu Q, et al. Interleukin-21 promotes germinal center reaction by skewing the follicular regulatory $T$ cell to follicular helper $T$ cell balance in autoimmune BXD2 mice. Arthritis Rheumatol. 2014;66:2601-12.

22. Lee SY, Jung YO, Ryu JG, et al. 2014. Intravenous immunoglobulin attenuates experimental autoimmune arthritis by inducing reciprocal regulation of Th17 and Treg cells in an interleukin-10dependent manner. Arthritis Rheumatol. 2014;66:1768-78.

23. Qin L, Waseem TC, Sahoo A, Bieerkehazhi S, Zhou H, Galkina EV, et al. Insights Into the Molecular Mechanisms of T Follicular Helper-Mediated Immunity and Pathology. Front Immunol. 2018;9:1884.

24. Rosenzwajg M, Lorenzon R, Cacoub P, Pham HP, Pitoiset F, et al. Immunological and clinical effects of low-dose interleukin-2 across 11 autoimmune diseases in a single, open clinical trial. Ann Rheum Dis. 2019;78:209-17.

25. Humrich JY, Spee-Mayer C, Siegert E, Bertolo M, Rose A, et al. Low-dose interleukin-2 therapy in refractory systemic lupus erythematosus: an investigator-initiated, single-centre phase 1 and 2a clinical trial. The Lancet Rheumatology. 2019;1(1):e44-54.

\section{Figures}




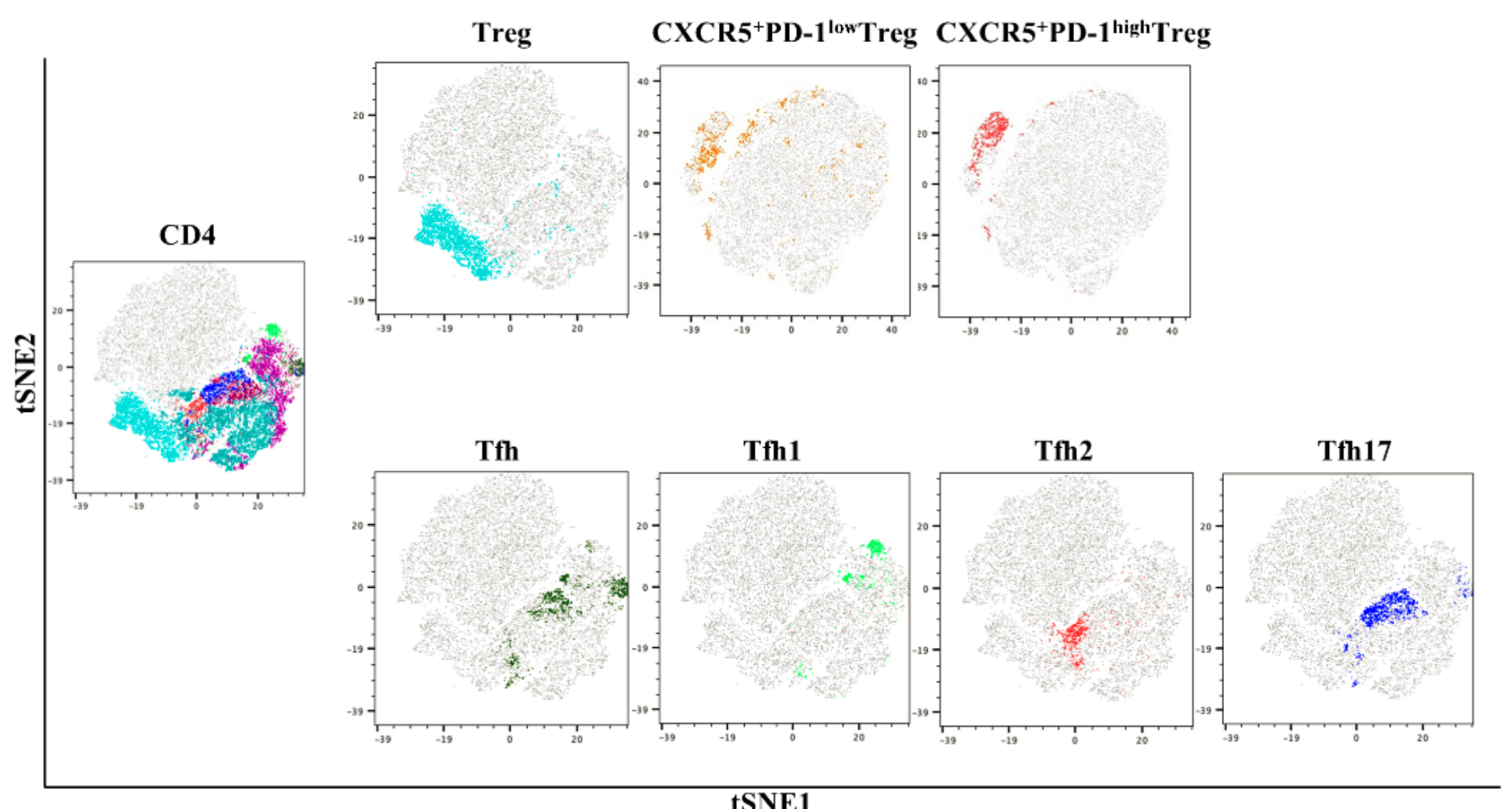

tSNE1

\section{Figure 1}

tSNE analysis of CD4 T subsets based on immune cells flow cytometry markers. tSNE, t-stochastic neighbor embedding. 


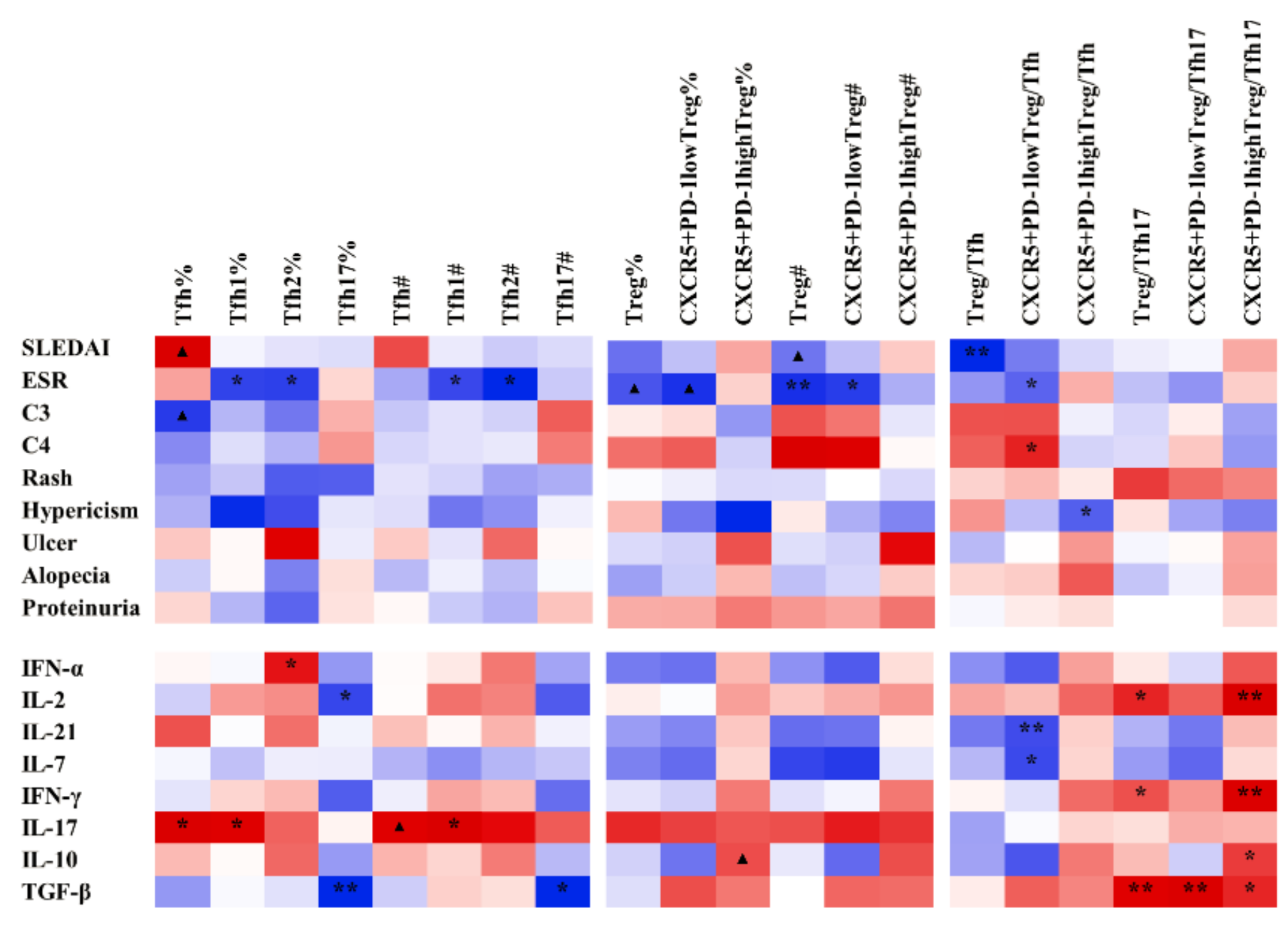

\section{Figure 2}

The correlations between CD4 T cell subsets and clinical characteristics in SLE patients. SLEDAI, SLE Disease Activity Index. ESR, erythrocyte sedimentation rate. C3, complement 3. C4, complement 4. IFN-a, interferon-a. IL-2, Interleukin-2. IL-21, Interleukin-21. IL-7, Interleukin-7. IFN- $\gamma$, interferon-ү. IL-17, Interleukin17. IL-10, Interleukin-10. TGF- $\beta$, tumor necrosis factor- $\beta .{ }^{* *}, P<0.01$. *, $P<0.05$. $\mathbf{\Lambda}, P<0.1$. \#, absolute number. \%, percentage in lymphocyte. 


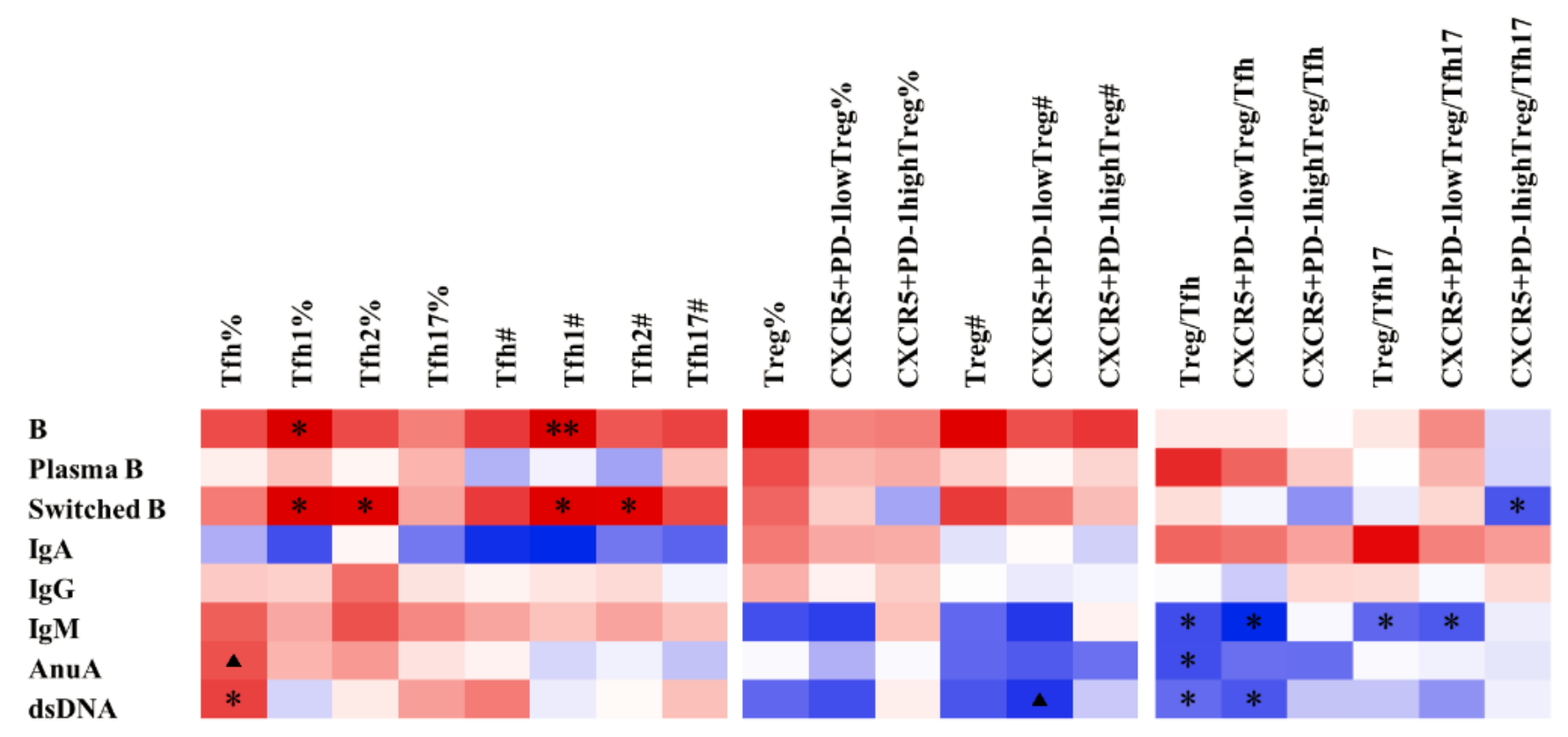

\section{Figure 3}

Correlations between CD 4 T cell subsets, B cells and immunoglobulins in SLE. B, B cell. Plasma B, Plasma B cell. Trans B, class-switched memory B cells. IgA, immunoglobulin A. IgG, immunoglobulin G. IgM, immunoglobulin M. ANuA, anti-nucleosome antibodies. dsDNA, Anti-double stranded DNA antibodies. ${ }^{*}, \mathrm{P}<0.05$. ${ }^{\star}, \mathrm{P}<0.01$. $\mathbf{\Delta}, \mathrm{P}<0.1$. \#, absolute number. \%, percentage in $\mathrm{LY}$. 
A

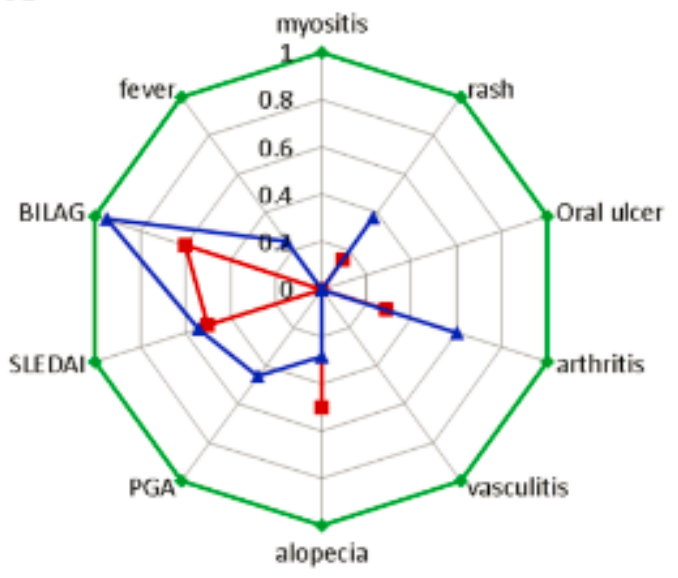

C

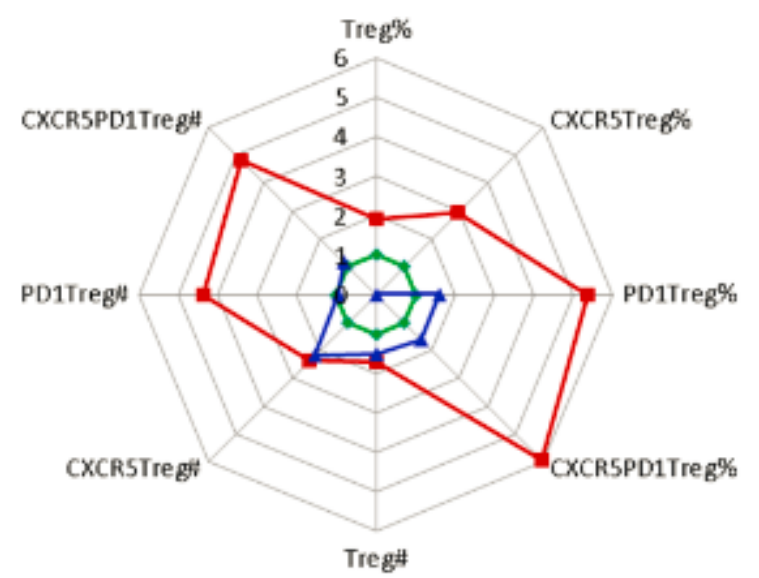

B

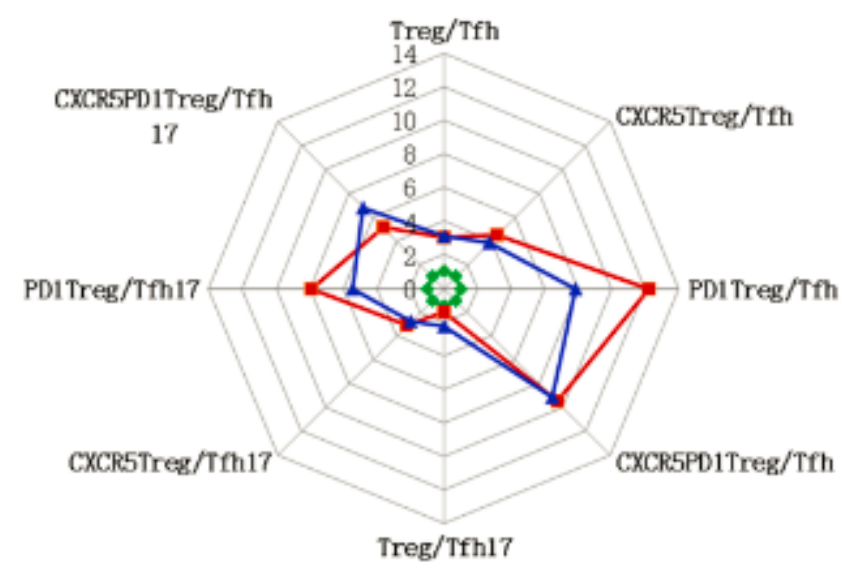

D

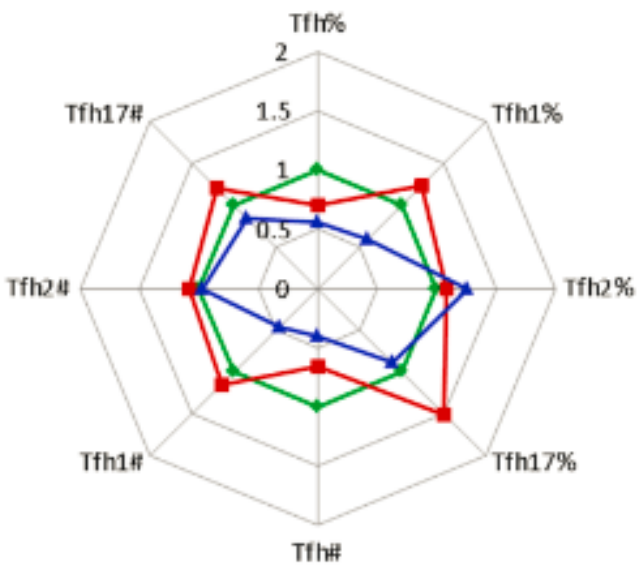

$\rightarrow$ Baseline $\rightarrow-1 L-2 \quad \mp$ traditional

\section{Figure 4}

The response of clinical and immune cells to Low-dose IL-2 $(n=30)$ and traditional $(n=30)$ therapy in SLE. (A) Relative change of disease activity value and patient number. (B) Relative change of ratios at baseline and week 10. (C) Relative change of Treg subsets at baseline and week 10. (D) Relative change of Tfh subsets at baseline and week 10. \#, absolute number. \%, proportion in lymphocyte. BILAG, British Isles Lupus Assessment Group. SLEDAI, Safety of Estrogens in Lupus Erythematosus National Assessment version of the SLE Disease Activity Index. SRI-4, SLE Responder Index-4. PGA, physician's global assessment.

\section{Supplementary Files}

This is a list of supplementary files associated with this preprint. Click to download. 
- supplementaryfigure2020.11.29.pptx 